\title{
DISTRIBUTION OF CONDUCTION VELOCITY (DCV) FROM MEASURED F- WAVE LATENCY FOR DETECTION OF CERVICAL SPONDYLOTIC RADICULOPATHY AND MYELOPATHY (CRM)
}

\author{
M Obaidur Rahman* and K Siddique-e Rabbani
}

Department of Biomedical Physics and Technology, University of Dhaka

Corresponding author email: mobaidur@yahoo.com

\begin{abstract}
In the previous work of our extended group at Dhaka, Distribution of Conduction Velocity (DCV) of motor nerve fibres in a peripheral nerve trunk was established as an approximate mirror image of Distribution of FLatency (DFL), for which consecutive 30 to 40 F-latencies were used through multiple stimulation of a peripheral nerve trunk. Using patterns of DFL a considerable amount of experience has been built up in the detection of Cervical Spondylotic Radiculopathy and Myelopathy (CRM). In the present work we have obtained DCV directly from the Conduction Velocity (CV) values obtained from each of the F-latencies. Because of the inverse relationship between latency and velocity, a small variation to the mirror image should be present, particularly when discrete bin widths are used to obtain the frequency distribution. Therefore, one challenge was to choose an appropriate bin width while obtaining DCV directly so that the patterns can be related to those of DFL easily for the detection of CRM. To obtain DFL our extended group at Dhaka had used a bin width of 2 ms which gave good results. To choose a corresponding bin width for DCV four recognized measures of central tendency: Average of the range, Median, Mode and Mean (weighted average) were used and the resulting patterns of DCV were compared to that of DFL to determine the percentage of matches with respect to the detection of CRM. It was observed that Median gave the best DCV with $89 \%$ matches followed closely by Mode giving $83 \%$ match. The other two gave much lower values, $67 \%$ and $50 \%$. Therefore, median value gave the best match which could be used to obtain DCV from the CV values directly, for the determination of CRM.
\end{abstract}

Key words: Distribution of F-Latency, Distribution of Conduction Velocity, Bin width, Radiculopathy, Myelopathy

\section{INTRODUCTION}

A peripheral nerve trunk has thousands of nerve fibres with different conduction velocities and their properties are adequately described through a statistical frequency distribution known as the Distribution of Conduction Velocity (DCV). For a proper assessment of neural health and diagnosis of neuropathy the DCV would have been an ideal parameter if it could be measured clinically. However, no established technique exists for the determination of DCV in a clinical setting. Our extended group at Dhaka University conceived and developed a simple method to obtain an approximate and relative DCV of motor nerve fibres in a peripheral nerve trunk as a mirror image of a new parameter, which was named by the group as Distribution of F-Latency (DFL).

F-latency is a standard parameter widely measured in an EMG clinic as a late evoked EMG potential obtained through artificial stimulation of a peripheral nerve (Magladery and McDougal 1950). For the median nerve, electrical stimulation is given at the wrist while the evoked potential from the Thenar muscle (APB muscle) at the base of the thumb is recorded using appropriate electrodes and instrumentation (Figure 1). The orthodromic (direction of the natural signal propagation) action potentials along all the motor fibres contribute to a large compound muscle action potential from the APB muscle and is called the M-wave. The antidromic (opposite to the direction of the natural signal propagation) action potentials travel to the respective cell bodies at the vertebral roots (anterior horn 
cell) where nothing happens in most of the motor neurons. However a few percent of the cell bodies of these motor neurons backfire with an estimated delay of about $1 \mathrm{~ms}$ (Kimura 1989) and then travels back to APB muscle causing a much smaller and delayed F-wave. Since the recruitment of motor neurons that contribute to the F-wave is random, different F-responses will have contributions from different motor nerve fibres with different conduction velocities. Therefore, the latencies, sizes and shapes of Fresponses will vary from event to event (for multiple stimulations) for the same nerve of the same subject. Based on the above observations our extended group had proposed that a statistical frequency distribution DFL of the F-latencies is basically a reflection of the DCV of the motor nerve fibres that contribute to overall F-responses and they had established this through experiments

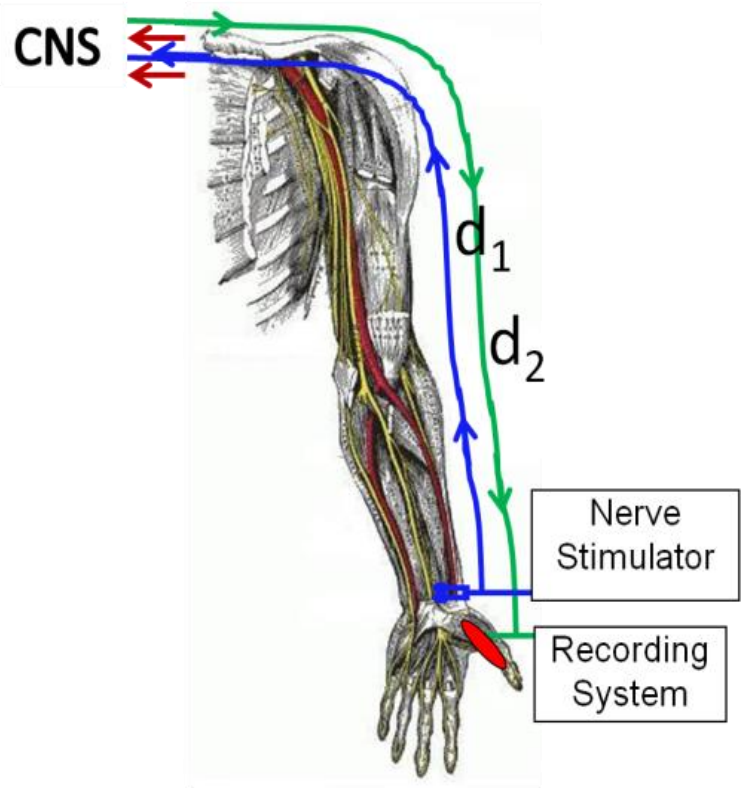

Figure 1: Stimulated signal travel through the median nerve on human subjects (Rabbani et al, 2007).

DFL involves obtaining multiple F-responses with a time interval of about $1 \mathrm{sec}$ between stimulations and then making a statistical frequency distribution of the F-latency values thus obtained. This long time delay was chosen so that the physical mechanisms involved due to a stimulation relax back their normal situations before the next stimulation. This would ensure that the physical mechanisms for each stimulation are independent of the previous event, or in other words, the events are random.

Furthermore, patterns of DFL have been related by our extended group to conditions of cervical radiculopathy (compression of the nerve roots) and myelopathy (compression of spinal cord), which we would refer to as Cervical spondylotic Radiculopathy and Myelopathy (CRM) in this work (Alam and Rabbani 2010). Further work on this aspect has established the hypothesis on a stronger footing and has established DFL as a viable method for detection of CRM (Hossain et al 2011, Rabbani 2011a, Rabbani 2011b, Rabbani et al 2014, Chowdhury 2013, Chowdhury et al 2014). DFL with a single sharp peak has been related to normalcy while a broad peak, double peak or a triple peak was related to CRM. The broad peak has again been defined as the one where the frequency value at a bin adjacent to the peak is equal or greater than one third of the peak value (Rabbani et al 2014). Therefore, the pattern is very subtle, particularly for such a broad peak, which is very close in pattern to a single peak.

Since a considerable experience has been established in relating DFL patterns to CRM, it was thought that if DCVs have similar patterns then this experience can be used in the detection of CRM employing the method of obtaining DCV being proposed in the present work. In other words, we would like to see similar patterns, although inverted as a mirror image, for DFL and DCV. However, because of the inverse relationship between latency and velocity, this mirror image is not exact, a small variation is expected, particularly when discrete bin widths and not too large sample sizes are employed to obtain the frequency distribution.

To obtain DFL, 30 to 40 consecutive F-latencies are usually obtained through multiple stimulation of a peripheral nerve trunk with adequate intervals between stimulations, typically about $1 \mathrm{sec}$, as mentioned before. 
In an earlier study Chowdhury (Chowdhury et al 2014) applied about 40 supramaximal electrical stimulations to the median nerve at the wrist in sequence, with intervals of one second between each to obtain about 30 or more F-responses from the corresponding thenar muscle of 31 subjects, from both sides. An excess number of stimulations was given to each nerve since not all stimulations give rise to F-responses. These data were obtained using nerve conduction equipment made by our extended group which has been in use in a clinic over many years. The muscle was kept relaxed during the experiment. The actual number of stimulations given varied somewhat depending on the cooperation of the subject. The time sequence of the collected F-latencies from each nerve was maintained and recorded. The measurements were carried out at room temperature, which remained steady at or around $30^{\circ} \mathrm{C}$.

In the present work it is intended to obtain DCV from individual conduction velocity $(\mathrm{CV})$ values that are obtained through converting the corresponding F-latencies. Data obtained experimentally by Chowdhury et al 2014 were used for the purpose. Then choosing an appropriate bin width, a frequency distribution of CV will be obtained which should be the desired DCV. Most of the DFLs obtained in our laboratory earlier used a bin width of $2 \mathrm{~ms}$ and a large experience has been built up on the analysis of its pattern for the detection of CRM as mentioned before (Rabbani et al 2007, Rabbani et al 2014, Chowdhury et al 2014). Therefore, we could use that experience if a corresponding bin width for DCV is chosen when analyzing individual CV values to obtain DCV, in order to obtain a similar, but mirror image pattern.

However, as mentioned before, the relationship between CV and Latency is non-linear therefore, to correspond to a fixed bin width of DFL, the bin width for CV cannot be kept constant over the whole range. This will make the analysis difficult and we would like to have a fixed bin width for DCV as well. Therefore, the aim of the present work is to determine an optimized bin width for obtaining DCV from individual CV values while keeping the pattern of the DCV as close as to the mirror image of DFL, to aid the detection of CRM.

\section{METHODOLOGY}

As mentioned before, the present work used the data collected earlier by our extended group (Chowdhury, 2013, Chowdhury et al., 2014). However, the nerve lengths of the subjects were not measured during that study. Out of the 31 subjects used in that study, only 10 were available during the present work whose nerve length could be measured. Besides, out of the 20 median nerves of the 10 subjects, F-latency data were available for 18 nerves only, which was used in the present analysis.

The data on the 18 median nerves came from 10 subjects, 1 female and 9 male, aged between 24 and 56 yrs with a mean of $33.25 \mathrm{yrs}$ ( $\pm 12.26 \mathrm{yrs}$ ). The lengths of the nerve pathway from the stimulation site at wrist to the vertebral roots and then back to the relevant muscle at the base of the thumb were measured for all the subjects. Of course, some uncertainty would be involved as the nerve pathway is not accessible directly from outside, some estimation had to be incorporated.

To analyse the latency and the conduction velocity involved in an F-wave let us consider a supramaximal stimulation applied to the median nerve at the wrist (Figure 2). The orthodromic (direction of the natural signal propagation) action potentials along all the motor fibres contribute to a large compound muscle action potential, generated from the APB muscle at the base of the thumb, called the M-wave. The antidromic (opposite to the direction of the natural signal propagation) action potentials travel to the respective cell bodies at the vertebral roots (anterior horn cell) where nothing happens in most of the neurons. However a few percent of the motor neurons backfire with an 
estimated delay of about $1 \mathrm{~ms}$ (Kimura 1989) and then travels back to APB muscle causing the much smaller and delayed F-wave.

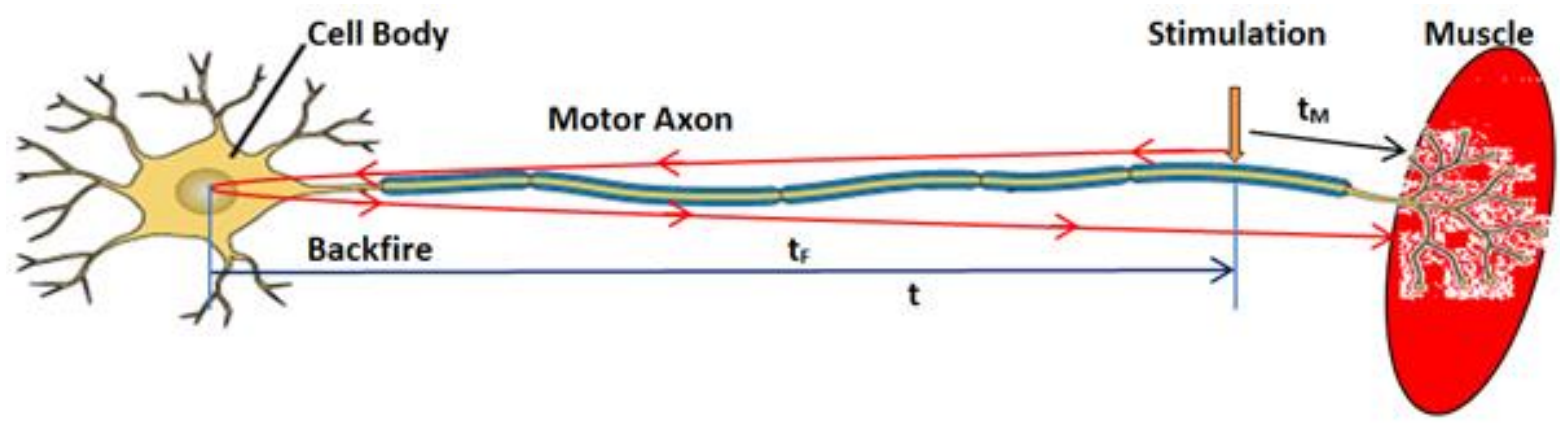

Figure 2: Schematic picture of nerve conduction due to artificial stimulation at a midpoint to illustrate latencies for $\mathrm{M}$-wave and $\mathrm{F}$-wave

We usually measure the latencies to the onset of the M-wave or the F-wave, and let these be represented by $t_{M}$ and $t_{F}$. Therefore, the time of travel ( $t$ ) from the wrist to the cell body in the spinal cord will be given by,

$$
\mathrm{t}=\left(\mathrm{t}_{\mathrm{F}}-\mathrm{t}_{\mathrm{M}}-1\right) / 2
$$

The division by 2 appears since the F-wave action potential travels the corresponding distance twice.

If this distance is $d$ then the Conduction velocity $C V$ of the fastest fibre in the particular F-wave is given by,

$$
C V=\frac{d}{t}
$$

This was given the name FWCV by Kimura (Kimura, 1989).

A relative DCV of such motor nerve fibres may be obtained by grouping them into a number of suitable bins in terms of velocity. However, as mentioned above,

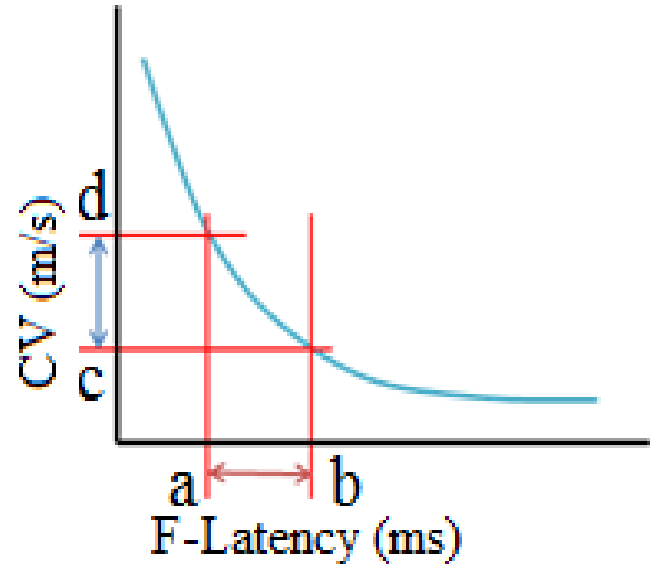

Figure 3: Non-linear curve of $\mathrm{CV}$ against $\mathrm{F}$ latency illustrates problem with bin-width we would like to optimize this choice of the bin width so that the pattern of DFL has close resemblance to the mirror image pattern of DFL, typically obtained using a bin width of $2 \mathrm{~ms}$. Since latency is inversely proportional to $\mathrm{CV}$, if bin widths for $\mathrm{DCV}$ are chosen to correspond to the constant F-latency bin over the whole range, varied bin widths for DCV will result, which is not a desired situation. This is illustrated in Figure 3. Suppose ab represents the bin width (shown exaggareted) for DFL. The corresponding bin width for DCV would be cd. Now as the bin location for DFL is shifted right or left keeping the range ab constant, the corresponding bin width for DCV will change.

However, when obtaining DCV from individual CV values, it is expected to use a fixed bin width. Therefore, in the present work we were looking for an optimized fixed bin width for DCV which would give a reasonable correspondence between the patterns of DFL and DCV for the same raw data of F-latencies. To elaborate this point, we wanted to fix the location of the bin group on the F-latency axis so that the corresponding bin width for DCV gives a reasonable pattern correspondence. With this aim four statistical parameters of the F-latency data were tried to identify the location of the bin group for DFL corresponding to which the bin width for DCV will be chosen. These are, for the range 
of non-zero values of F-latency, i) average of the minimum and maximum, ii) median, iii) mode and iv) weighted average. For the weighted average, the number of times a given F-latency repeated was considered for the average appropriately.

The corresponding bin widths for DCV using the above four parameters were obtained as follows. For the first parameter, we took the average of the maximum and minimum values of measured FLatencies, which we denote by X. Similarly we took the average of the corresponding maximum and minimum $\mathrm{CV}$ values and denote this by $\mathrm{Y}$. The desired bin width $\left(\mathrm{W}_{\mathrm{V}}\right)$ for DCV to correspond to a bin width $\mathrm{W}_{\mathrm{L}}$ of DFL (which is $2 \mathrm{~ms}$ for our study) is obtained using the formula,

$$
W_{V}=\frac{Y}{X} \times W_{L}
$$

In a similar way replacing $\mathrm{X}$ and $\mathrm{Y}$ with the corresponding median, mode and weighted average values, we obtained the corresponding bin widths for DCV using the other three parameters.

Following the above methods we chose individual bin widths to generate DCVs for each of the 18 nerves based on the above four parameters and then tried to match the DFL and DCV curves visually. As indicated before, these curves are expected to be approximate mirror images of each other, and we looked for pattern matching in view of the above.

Then we tried to compare the performances of the four chosen parameters. For this we studied each DCV pattern and the corresponding DFL pattern. If the DCV pattern appeared to be a good match of the mirror image pattern of the respective DFL we tagged it with a ' $Y$ ' for 'yes'. The ones that did not match were tagged ' $\mathrm{N}$ ' for 'no'. The results were compiled in a table and the total number of correct matches (' $Y$ ') were counted corresponding to each of the four parameters. This value was used as a numerical figure to describe the quality of match for a particular method of obtaining the bin width for DCV. The parameter giving the maximum match was chosen as the one to use for obtaining bin width.

Finally, the mean of the chosen bin widths of the 18 nerves tested was obtained to give a reasonable value of bin width for DCV, expecting that this would give the best match for all other subjects too.

\section{RESULTS}

Table 1 shows the individual bin widths calculated for DCV based on the four parameters described before for all the 18 nerves under study, with a fixed $2 \mathrm{~ms}$ bin width for DFL. It also shows the number of F-responses obtained from the particular nerve and an estimation of the nerve length. Figure 4 shows a few representative DFLs and corresponding DCVs using bin widths calculated based on the four parameters respectively. The results of the visual matching of the DCV patterns with the corresponding DFL patterns are given in Table 2 ( $\mathrm{Y}$ for a match, $\mathrm{N}$ for a non-match). The number of good matches for each of the four parameters was summed and the results are shown in Table 3. 


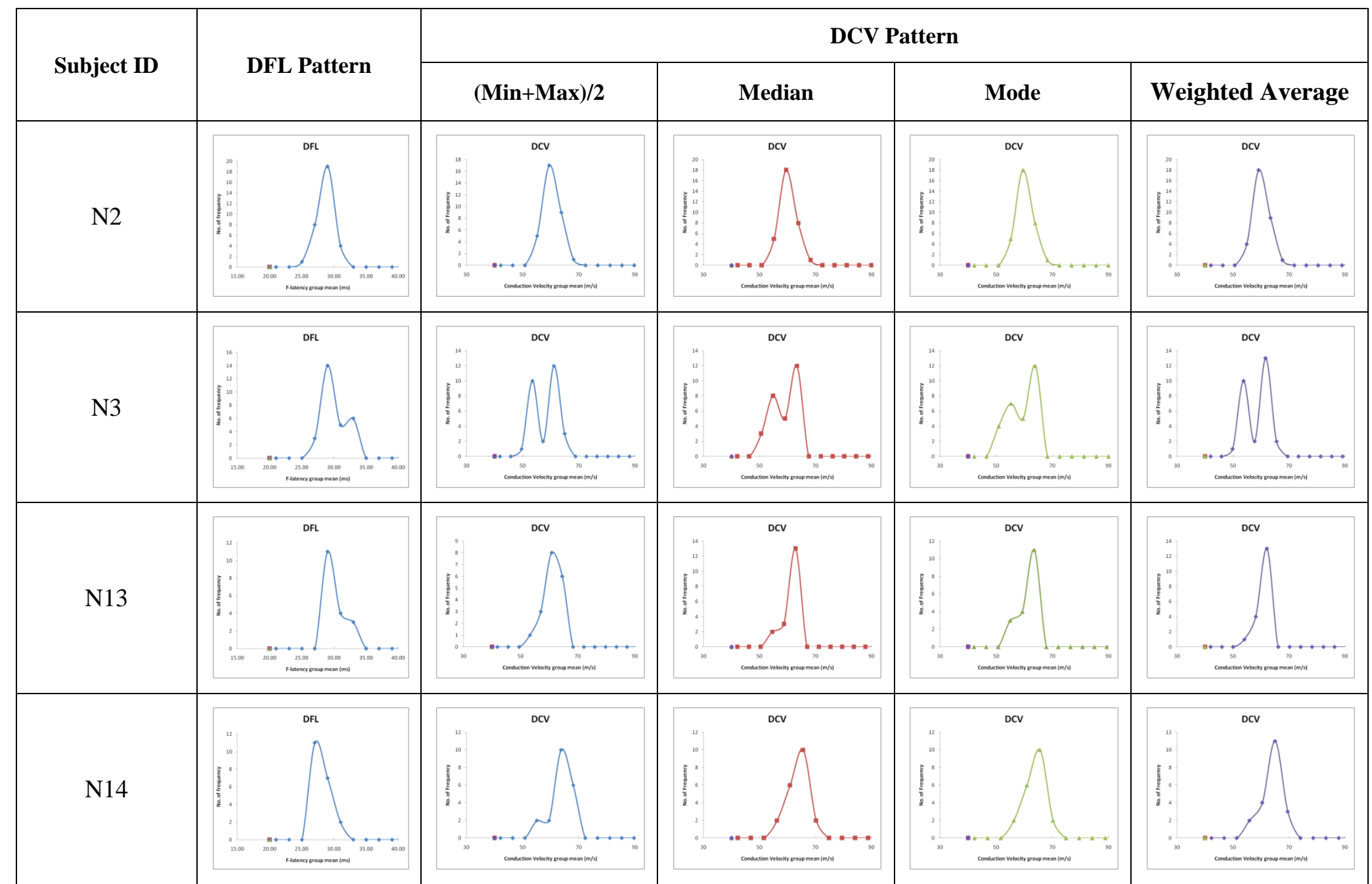

Figure 4: A few representative samples of DFL and DCV, the latter obtained using four parameters to calculate bin width. The results matching for these can be seen in Table 2, which gives a guide to the decision making process. 
Table 1: Bin widths for DCV to correspond to a bin width of $2 \mathrm{~ms}$ for DFL

\begin{tabular}{|c|c|c|c|c|c|c|}
\hline \multirow{2}{*}{$\begin{array}{l}\text { Subj } \\
\text { ect } \\
I D\end{array}$} & \multirow{2}{*}{$\begin{array}{c}\text { No of } \\
\text { F-responses }\end{array}$} & \multirow{2}{*}{$\begin{array}{l}\text { Nerve } \\
\text { Length } \\
(\mathrm{cm})\end{array}$} & \multicolumn{4}{|c|}{ DCV Bin width, in $\mathrm{m} / \mathrm{s}$} \\
\hline & & & $\frac{(\operatorname{Min}+\operatorname{Max})}{2}$ & Median & Mode & Average \\
\hline N1 & 30 & 156 & 5.41 & 5.47 & 4.51 & 5.40 \\
\hline $\mathrm{N} 2$ & 32 & 140 & 4.32 & 4.34 & 4.34 & 4.25 \\
\hline N3 & 28 & 142 & 3.84 & 4.25 & 4.35 & 3.93 \\
\hline N4 & 35 & 168 & 4.06 & 4.11 & 4.17 & 4.07 \\
\hline N5 & 22 & 170 & 4.68 & 4.63 & 4.63 & 4.60 \\
\hline N6 & 25 & 146 & 4.54 & 4.39 & 4.83 & 4.50 \\
\hline N7 & 15 & 146 & 4.14 & 4.17 & 4.17 & 4.19 \\
\hline N8 & 35 & 144 & 5.35 & 5.24 & 5.24 & 5.31 \\
\hline N9 & 34 & 140 & 4.90 & 4.78 & 4.78 & 4.89 \\
\hline N10 & 26 & 156 & 5.02 & 5.03 & 4.91 & 5.10 \\
\hline N11 & 27 & 156 & 4.73 & 4.91 & 4.91 & 4.88 \\
\hline N12 & 27 & 150 & 3.88 & 3.69 & 3.69 & 3.81 \\
\hline N13 & 18 & 148 & 3.78 & 4.16 & 4.28 & 4.02 \\
\hline N14 & 20 & 150 & 4.31 & 4.65 & 4.65 & 4.54 \\
\hline N15 & 32 & 156 & 4.71 & 5.02 & 4.94 & 5.06 \\
\hline N16 & 38 & 150 & 5.53 & 5.56 & 6.07 & 5.56 \\
\hline N17 & 31 & 144 & 4.98 & 4.82 & 4.82 & 5.06 \\
\hline N18 & 36 & 150 & 4.88 & 5.08 & 5.21 & 5.03 \\
\hline
\end{tabular}

Table 2: Agreement of DCV patterns with corresponding mirror image of DFL patter, visual assessment. (Y for a match, $\mathrm{N}$ for a non-match)

\begin{tabular}{ccccc}
\hline $\begin{array}{c}\text { Subj } \\
\text { ID }\end{array}$ & \multicolumn{4}{c}{ DCV pattern matched with DFL pattern } \\
(Min + Max $)$ & Median & Mode & Average \\
\hline N1 & Y & Y & Y & Y \\
N2 & Y & Y & Y & Y \\
N3 & N & Y & Y & N \\
N4 & Y & Y & Y & Y \\
N5 & N & Y & Y & Y \\
N6 & Y & Y & Y & Y \\
N7 & N & Y & Y & Y \\
N8 & Y & N & N & Y \\
N9 & Y & Y & Y & Y \\
\hline
\end{tabular}

\begin{tabular}{ccccc}
\hline $\begin{array}{c}\text { Subj } \\
\text { ID }\end{array}$ & \multicolumn{4}{c}{ DCV pattern matched with DFL pattern } \\
(Max $)$ & Median & Mode & Average \\
\hline N10 & N & N & N & N \\
N11 & N & Y & Y & Y \\
N12 & Y & Y & Y & N \\
N13 & N & Y & Y & N \\
N14 & N & Y & Y & N \\
N15 & N & Y & Y & Y \\
N16 & Y & Y & N & Y \\
N17 & N & Y & Y & N \\
N18 & Y & Y & Y & Y \\
\hline
\end{tabular}


Table 3: Number of DCV pattern considered as a mirror of standard DFL pattern through visual assessment

\begin{tabular}{|c|c|c|c|c|}
\hline & $\frac{(\operatorname{Min}+\operatorname{Max})}{2}$ & Median & Mode & Average \\
\hline $\begin{array}{l}\text { Number of DFL } \\
\text { Pattern }\end{array}$ & 9 & 16 & 15 & 12 \\
\hline $\begin{array}{l}\text { Percentage of } \\
\text { total }\end{array}$ & 50 & 89 & 83 & 67 \\
\hline
\end{tabular}

It can be seen that the median based bin width gave the best match, at $89 \%$ ( 16 out of 18 nerves).

Finally, the mean of the bin widths for the parameter 'median' to give the best match to DFLs with $2 \mathrm{~ms}$ bin width was found to be $4.7 \mathrm{~m} / \mathrm{s}$ (standard deviation: $0.5 \mathrm{~m} / \mathrm{s}$ ).

\section{DISCUSSIONS}

Investigation of nerve conduction are being carried out for quite a long time, but nothing more than the onset latency of evoked potentials have practical applications in quantitative evaluation till now. This diagnosis gives information only about the fastest group of nerve fibers while many diseases or disorders involve loss or slowing of conduction of the middle and slow nerve fibers which do not get detected in the existing methods. Therefore, the best diagnostic method would be one that gives a complete distribution of conduction velocity or DCV of a nerve trunk. The evoked potential waveshapes are expected to contain this information, but reproducible techniques are yet to be developed to extract the information, as desired.

As DFL owes its origin to DCV of motor nerve fibers as has been established through earlier work of our extended group, it was also suggested that an approximate relative DCV of motor nerves can be obtained directly as a mirror image of DFL. However, we wanted to obtain DCV directly from conduction velocity $(\mathrm{CV})$ values obtained from each of the multiple F-responses used to obtain DFL. Since DFL have been used for quite some time in identifying cervical spondylotic radiculopathy or myelopathy (CRM), a significant amount of experience has been accrued on the patterns of DFL. Therefore, our objective was to get a good match of the patterns of the mirror image of DFL and DCV. A challenge is the choice of a constant bin width for DCV as this choice can change the pattern of the distribution significantly if the total number of events (F-responses) are not too many. The objective of this paper was to make a study that would help in choosing an appropriate bin width for obtaining the desired patterns of DCV using the above method.

Since our extended group has been using $2 \mathrm{~ms}$ bin width to obtain DFL from 30 to 40 F-responses we wanted to find the most appropriate bin width to correspond to the above choice when obtaining DCV from the F-wave conduction velocity values.

In Table 1 it was observed that to correspond to $2 \mathrm{~ms}$ bin width for DFL the bin width for conduction velocity varies somewhat for different subjects depending on what formulae we choose to obtain the bin width for DCV. The nerve length also played a role. Table 2 shows the quality of match between DFL and DCV for all the nerves tested. Table 3 shows the percentage of nerves for which a good match was obtained, using the four different bases for bin width calculation. It shows that out of 18 nerves, the best match was obtained for the median based bin width, at $89 \%$. The next was for mode, at $83 \%$ while the other two based on averages are much less, at $50 \%$ and $67 \%$ respectively. This 
observation suggests that bin width calculation based on the median could be used to obtain an acceptable DCV pattern that would match with the corresponding DFL patterns.

Again, as a result of the present work we can say that the mean of the bin widths for the parameter 'median' to give the best match to DFLs with $2 \mathrm{~ms}$ bin width is $4.7 \mathrm{~m} / \mathrm{s}$. Therefore, this value can be used for obtaining DCVs for nerves of the upper limb (median and ulnar) from conduction velocity values obtained using multiple F-responses.

One of the major limitations of this study was the measurement of nerve length. The median nerve originates from the lateral and medial cords of the brachial plexus, and has contributions from ventral roots of C5, C6 \& C7 (lateral cord) and C8 \& T1 (medial cord) and passes through the carpal tunnel. The ones that are relevant for the present work were those derived from C7, C8 and T1 (Alam and Rabbani 2010). It is impossible to measure the nerve length accurately, besides, those from the three roots will differ in length. Therefore, an approximate estimation was done with the help of anatomical reference points. However, an uncertainty of about $5 \mathrm{~cm}$ will lead to an error of about $3 \%$ since the total path length is of the order of $150 \mathrm{~cm}$, which is negligible in this type of study.

It needs to be mentioned that a sample size of about 30 to 40 gives a representative distribution of a large population but these distributions will have a certain amount of uncertainty. The uncertainties are reduced if the sample sizes are made larger. In our case we cannot increase the sample size very much since it is uncomfortable and therefore unacceptable to patients. Therefore, we have to try to get the best from a sample size of 30 to 40 F-latencies.

The main aim of the present work was to obtain DCVs from multiple F-responses and to use their patterns in detecting cervical radiculopathy or myelopathy, which seems to work well, in comparison to the established patterns of DFL. Since both DFL and DCV are statistical techniques, some uncertainty will remain. Therefore, these methods are best to be used for screening of patients, those found positive will be required to go through further investigation. However, even the outcome of DFL or DCV may be improved through the incorporation of some simple clinical tests carried out by Neurologists when the outcome of DFL or DCV will be very important.

\section{ACKNOWLEDGEMENT}

Ministry of Science and Technology, Government of the People's Republic of Bangladesh and International Science Program of Uppsala University, Sweden for part support. Acknowledgements are also due to Dr. Ehsan Alam Chowdhury, for helping in measuring nerve lengths through locating anatomical landmarks on the subjects' body.

\section{REFERENCES}

Alam MJ and Rabbani KS, 2010, Possible detection of cervical spondylotic neuropathy using Distribution of F-latency (DFL), a new neurophysilogical parameter. BMC Research Notes, 3:112.

Chowdhury EA, 2013, Study of the efficacy of the Distribution of F-latency (DFL) in the diagnosis of cervical spondylosis. M.Phil. Thesis, University of Dhaka.

Chowdhury EA, Rahman MO and Rabbani KS, 2014, Comparing DFL and MRI findings. Bangladesh Journal of Medical Physics, 7, 46-55. 
Hossain MI, Chowdhury EA, Mamun AA, Salam A, Baig TN and Rabbani KS, 2011, Use of Distribution of F-Latency (DFL) in the detection of cervical spondylotic neuropathy, Bangladesh Journal of Medical Physics, 4, 37-42.

Kimura J, 1989, Electrodiagnosis in diseases of nerve and muscle: Principles and practice, Philadelphia, F.A. Davis company.

Magladery JW, Mcdougal DB, 1950, Electrophysiological Studies of nerve and reflex in normal man. I. Identification of certain reflexes in the electromyogram and the conduction velocity of peripheral ischemia. Bull Johns Hopkins Hosp, 86, 265-290.

Rabbani KS, 2011a, Distribution of F-Latency (DFL) - a new nerve conduction parameter Recent developments and possibilities. Biomedical Engineering, Editors - Ramesh R. Galigekere et. al., Narosa Publishing House, India, 137-142.

Rabbani KS, 2011b, Hypotheses to Explain the Occurrence of Multiple Peaks of DFL in Nerve Conduction Measurement. Bangladesh Journal of Medical Physics, 4, 27-36.

Rabbani KS, 2012, Neuro-physiological measurements for diagnosis using evoked responses. Bangladesh Journal of Med Phys, 5, 1-24.

Rabbani KS, Alam MJ and Salam A, 2007, Frequency Distribution of F-Latencies (DFL) has Physiological Significance and Gives Distribution of Conduction Velocity (DCV) of Motor Nerve Fibres With Implications for Diagnosis. J. Biol. Phys., 33, 291-303.

Rabbani KS, Yassin N and Lo YL, 2014, Identification of Cervical Spondylotic Radiculo-Myelopathy using Distribution of F-Latency (DFL), a new nerve conduction parameter. Bangladesh Journal of Med Phys, 7, 34-45. 